\title{
Alkylated albumin-derived dipeptide C(-HETE)P derivatized by propionic anhydride as a biomarker for the verification of poisoning with sulfur mustard
}

\author{
Annika Richter $^{1} \cdot$ Markus Siegert $^{1,2} \cdot$ Horst Thiermann ${ }^{2} \cdot$ Harald John $^{2}$ \\ Received: 29 April 2021 / Revised: 25 May 2021 / Accepted: 4 June 2021 / Published online: 2 July 2021 \\ (C) The Author(s) 2021
}

\begin{abstract}
Sulfur mustard (SM) is a banned chemical warfare agent recently used in the Syrian Arab Republic conflict causing erythema and blisters characterized by complicated and delayed wound healing. For medical and legal reasons, the proof of exposure to SM is of high toxicological and forensic relevance. SM reacts with endogenous human serum albumin (HSA adducts) alkylating the thiol group of the cysteine residue $\mathrm{C}^{34}$, thus causing the addition of the hydroxyethylthioethyl (HETE) moiety. Following proteolysis with pronase, the biomarker dipeptide C(-HETE)P is produced. To expand the possibilities for verification of exposure, we herein introduce a novel biomarker produced from that alkylated dipeptide by derivatization with propionic anhydride inducing the selective propionylation of the N-terminus yielding PA-C(-HETE)P. Quantitative derivatization is carried out at room temperature in aqueous buffer within $10 \mathrm{~s}$. The biomarker was found to be stable in the autosampler at $15^{\circ} \mathrm{C}$ for at least $24 \mathrm{~h}$, thus documenting its suitability even for larger sets of samples. Selective and sensitive detection is done by micro liquid chromatography-electrospray ionization tandem-mass spectrometry ( $\mu$ LC-ESI MS/MS) operating in the selected reaction monitoring (SRM) mode detecting product ions of the single protonated PA-C(-HETE)P (m/z 379.1) at m/z 116.1, m/z 137.0, and m/z 105.0. The lower limit of detection corresponds to $32 \mathrm{nM}$ SM in plasma in vitro and the limit of identification to $160 \mathrm{nM}$. The applicability to real exposure scenarios was proven by analyzing samples from the Middle East confirming poisoning with SM.
\end{abstract}

Keywords Biomarker $\cdot$ Chemical warfare agent $\cdot$ HETE moiety $\cdot$ Protein adduct $\cdot$ Propionic anhydride $\cdot$ Verification

\section{Introduction}

Sulfur mustard (SM, bis(2-chloroethyl)-sulfide) is a chemical warfare agent (CWA) and belongs to the class of vesicants $[1$, 2]. It has been used for more than 100 years with its first usage in 1917 during World War I in Ypres (Belgium) and most recently during the Syrian Arab Republic conflict [3-5]. Skin areas exposed to SM may develop erythema and painful blisters that are characterized by complicated and delayed wound healing [6]. The use, stockpiling, and production of SM is prohibited by the Chemical Weapons Convention (CWC) implemented by the Organisation for the Prohibition

Harald John

HaraldJohn@bundeswehr.org

1 Department of Chemistry, Humboldt-Universität zu Berlin, Brook-Taylor-Straße 2, 12489 Berlin, Germany

2 Bundeswehr Institute of Pharmacology and Toxicology, Neuherbergstrasse 11, 80937 Munich, Germany of Chemical Weapons (OPCW) [7]. Nevertheless, exposure to SM still represents a threat to the military as well as to the civilian population $[8,9]$. Therefore, reliable, rapid, simple, selective, and sensitive bioanalytical methods are demanded for the verification of SM exposure and to provide evidence for the violation of the CWC [7].

In vivo, SM can either be hydrolyzed to the non-toxic thiodiglycol (TDG) (detectable in urine for up to 1 week after exposure) or it may alkylate nucleophiles in, e.g., DNA and proteins like keratin, hemoglobin, or human serum albumin (HSA) [10-13]. These protein adducts represent long-lived analytical targets of SM exposure (half-life of HSA adduct at $\mathrm{Cys}^{34}: 22$ days) [14, 15]. All adducts are characterized by the presence of the SM-derived hydroxyethylthioethyl (HETE) moiety attached to nucleophilic sites of the protein $[12,14]$. Enzymatic cleavage of the HETE-HSA adduct with proteinase $\mathrm{K}$ generates the alkylated tripeptide $\mathrm{C}(-\mathrm{HETE}) \mathrm{PF}$ [14] and cleavage with pronase produces the alkylated dipeptide C(-HETE)P [16-18] that may be monitored by micro liquid chromatography-electrospray ionization 
tandem-mass spectrometry ( $\mu \mathrm{LC}$-ESI MS/MS). These peptide biomarkers have been successfully used in interlaboratory exercises of the OPCW [18] as well as to investigate real case plasma samples drawn in the Middle East in 2015 [4].

In principle, the extension of the toolbox for verification methods is a core requirement to optimize established methods or include alternative analytical targets. Accordingly, in 2019, Chen et al. introduced a method to derivatize the C(-HETE)P biomarker with benzyl chloroformate (BCF) [19]. This procedure prolongs the time for sample preparation significantly $(2 \mathrm{~h}$ of derivatization at $4{ }^{\circ} \mathrm{C}$ ) and makes use of the toxic and environmentally harmful $\mathrm{BCF}$. In addition, the derivatization yield was reported to be as low as $47 \%$, thus reducing the detectable amount of the biomarker [19]. Furthermore, no application to real cases of SM poisoning has been reported so far. Avoiding these restrictions, we herein present a novel, rapid, and quantitative method to propionylate $\mathrm{C}(-\mathrm{HETE}) \mathrm{P}$ at its N-terminus with the use of propionic anhydride and subsequent $\mu \mathrm{LC}$-ESI MS/MS analysis. The biomarker was characterized, compared to already established biomarkers and successfully applied to plasma samples from real cases of SM intoxication [4].

\section{Materials and methods}

\section{Chemicals and reagents}

Acetone (p.A.), acetonitrile (ACN, LC-MS grade), dioxane (99.8\%), isopropanol (iPrOH, LC-MS grade), and water (LiChrosolv) were purchased from Merck (Darmstadt, Germany). Formic acid (FA, $\geq 98 \%$ ) and $\mathrm{NaOCl}$ solution for decontamination $\left(12 \% \mathrm{Cl}_{2}\right)$ were from Carl Roth (Karlsruhe, Germany). Propionic anhydride ( $\geq 99 \%)$, trifluoroacetic acid (TFA, $\geq 99 \%$ ), triethylamine (TEA, $\geq 99.5 \%$ ) and phosphatebuffered saline (PBS) were from Sigma-Aldrich (Steinheim, Germany), pronase from Streptomyces griseus from Roche (lot no. 70327222, Mannheim, Germany), $\mathrm{NH}_{4} \mathrm{HCO}_{3}$ (ultragrade, $\geq 99.5 \%$ ) from Fluka (Buchs, Switzerland), and threefold deuterated atropine $\left(\mathrm{d}_{3}\right.$-Atr) from CDN Isotopes (Pointe Claire, Quebec, Canada). The $\mathrm{d}_{3}$-Atr working solution (3 ng/ $\mathrm{mL}$ ) was prepared in $0.5 \% \mathrm{v} / \mathrm{v}$ FA. Human ethylenediaminetetraacetic acid (EDTA) plasma from different individuals was purchased from in.vent Diagnostica (Hennigsdorf, Germany). SM was made available by the German Ministry of Defense and tested for integrity and purity in-house by nuclear magnetic resonance (NMR) spectroscopy.

\section{Incubation of plasma with SM}

EDTA-plasma $(980 \mu \mathrm{L})$ was mixed with SM solution $(20 \mu \mathrm{L}$ in $\mathrm{iPrOH}$ ) and incubated for $2 \mathrm{~h}$ at $37^{\circ} \mathrm{C}$ under gentle shaking. $\mathrm{SM}$ concentrations in plasma of $50 \mu \mathrm{M}$ and $12.5 \mu \mathrm{M}$ were used to produce references. Additional concentrations of SM were used as described below (section: linear range, limit of detection, and limit of identification). Blanks were incubated with $20 \mu \mathrm{L}$ iPrOH only. Samples were stored if necessary at $-20{ }^{\circ} \mathrm{C}$ until further processing.

\section{Precipitation of proteins from EDTA-plasma samples}

EDTA-plasma $(50 \mu \mathrm{L})$ was mixed with acetone $(250 \mu \mathrm{L})$ in a $1.5 \mathrm{~mL}$ reaction vial, vortex mixed for $10 \mathrm{~s}$ and centrifuged afterwards $(3500 \mathrm{~g}, 3 \mathrm{~min}$ at room temperature, RT). The supernatant was removed and the protein pellet washed with acetone $(250 \mu \mathrm{L})$ by sonication for $2 \mathrm{~min}$. After centrifugation $(3500 \mathrm{~g}, 3 \mathrm{~min}, \mathrm{RT})$ and removal of the supernatant, the pellet was air-dried prior to proteolysis.

\section{Proteolysis of the albumin adduct with pronase}

The protein pellet obtained from EDTA-plasma was suspended in $50 \mathrm{mM} \mathrm{NH}_{4} \mathrm{HCO}_{3}(200 \mu \mathrm{L})$. Pronase solution $\left(50 \mu \mathrm{L}, 10 \mathrm{mg} / \mathrm{mL}\right.$ in $50 \mathrm{mM} \mathrm{NH}_{4} \mathrm{HCO}_{3}$ ) was added prior to incubation for $2 \mathrm{~h}$ at $50^{\circ} \mathrm{C}$. Afterwards, ACN $(750 \mu \mathrm{L})$ was added followed by centrifugation $(13,000 \mathrm{~g}, 5 \mathrm{~min}, \mathrm{RT})$. The supernatant was removed, dried by vacuum, and redissolved in $50 \mathrm{mM} \mathrm{NH}_{4} \mathrm{HCO}_{3}(250 \mu \mathrm{L})$. An aliquot $(50 \mu \mathrm{L})$ was mixed with $10 \mu \mathrm{L}$ water and diluted $1: 3$ with $\mathrm{d}_{3}$-Atr working solution as an internal standard (IS) for subsequent $\mu \mathrm{LC}$-ESI MS/MS analysis in the selected reaction monitoring (SRM) mode (sample volume $20 \mu \mathrm{L}$ ). Another $50 \mu \mathrm{L}$ aliquot was subjected to derivatization.

\section{Standard protocol for the derivatization of C(-HETE)P with propionic anhydride}

A portion $(50 \mu \mathrm{L})$ of the redissolved dried supernatant after proteolysis containing C(-HETE)P was mixed with propionic anhydride $(10 \mu \mathrm{L}, \mathrm{RT})$ in a $0.5-\mathrm{mL}$ reaction vial and vortex mixed for $10 \mathrm{~s}$. An aliquot $(30 \mu \mathrm{L})$ of the analyte solution was diluted 1:3 with $\mathrm{d}_{3}$-Atr working solution for subsequent analysis of $20 \mu \mathrm{L}$ by $\mu \mathrm{LC}$-ESI high-resolution tandem-mass spectrometry (MS/HRMS) in the product ion scan (PIS) mode and by $\mu \mathrm{LC}$-ESI MS/MS in PIS and SRM mode.

\section{Optimization of the derivatization conditions}

The derivatization reaction was optimized with respect to the amount of propionic anhydride, reaction time, and water amount to stop the reaction (quenching). A portion $(50 \mu \mathrm{L})$ of the redissolved dried supernatant after proteolysis of reference $(50 \mu \mathrm{M}$ SM) plasma proteins containing C(-HETE)P was used for each experiment. Peak areas obtained from the extracted ion chromatogram (XIC) of the qualifying ion at $\mathrm{m} / \mathrm{z} 116.1$ (Qual 1) of PA-C(-HETE)P were determined. The 
amount of propionic anhydride was varied using $3 \mu \mathrm{L}, 5 \mu \mathrm{L}$, $10 \mu \mathrm{L}, 25 \mu \mathrm{L}$, and $50 \mu \mathrm{L}$. The reaction time was varied and stopped by quenching the reaction mixture with $125 \mu \mathrm{L}$ water after $10 \mathrm{~s}, 30 \mathrm{~s}, 1 \mathrm{~min}, 2 \mathrm{~min}, 3 \mathrm{~min}$, and $5 \mathrm{~min}$ of derivatization.

\section{Derivatization of C(-HETE)P with BCF}

The derivatization was carried out according to Chen et al. [19]. An aliquot $(50 \mu \mathrm{L})$ of the redissolved dried supernatant after proteolysis of reference $(50 \mu \mathrm{M} \mathrm{SM})$ plasma proteins was transferred into a $0.5-\mathrm{mL}$ reaction vial, cooled to $4{ }^{\circ} \mathrm{C}$ for $5 \mathrm{~min}$, and subsequently mixed with BCF $(5 \mu \mathrm{L}$, $200 \mathrm{mg} / \mathrm{mL}$ in dioxane, $4{ }^{\circ} \mathrm{C}$ ). The solution was incubated for $30 \mathrm{~min}$ at $4{ }^{\circ} \mathrm{C}$ under gentle shaking. The derivatization was terminated by the addition of $2 \mu \mathrm{L}$ TFA $(20 \% \mathrm{v} / \mathrm{v})$ and $3 \mu \mathrm{L} \mathrm{H}_{2} \mathrm{O}$. An aliquot $(30 \mu \mathrm{L})$ was diluted $1: 3$ with $_{\mathrm{d}_{3}}$-Atr working solution for subsequent $\mu \mathrm{LC}$-ESI MS/MS (SRM) analysis.

\section{$\mu L C-E S I$ MS/HRMS (PIS) analysis}

Analysis by MS/HRMS was carried out to determine the accurate masses of product ions using the PIS mode. For chromatographic separation, a micro LC 200 system (Eksigent Technologies, Dublin, CA, USA) equipped with a HTC-xt DLW autosampler (CTC Analytics, Zwingen, Switzerland) and a $20-\mu \mathrm{L}$ sample loop (Sunchrom, Friedrichsdorf, Germany) were used. Peptides were separated at $60^{\circ} \mathrm{C}$ on an Acquity UPLC HSS T3 column $(50 \times 1.0 \mathrm{~mm}$ I.D., $1.8 \mu \mathrm{m}, 100 \AA$; Waters, Eschborn, Germany) protected by a precolumn (Security Guard Ultra cartridge C18 peptide; Phenomenex, Aschaffenburg, Germany) applying a binary gradient mobile phase with $30 \mu \mathrm{L} / \mathrm{min}$ of solvent A $(0.05 \%$ $\mathrm{v} / \mathrm{v}$ FA) and solvent $\mathrm{B}\left(\mathrm{ACN} / \mathrm{H}_{2} \mathrm{O} 80: 20 \mathrm{v} / \mathrm{v}, 0.05 \% \mathrm{v} / \mathrm{v} \mathrm{FA}\right)$ : $\mathrm{t}$ [min]/B [\%]: 0/2, 11/30, 11.5/95, 13.5/95, 14/2, 15/2.

The $\mu \mathrm{LC}$ system was coupled via an ESI interface (Turbo $\mathrm{V}$ source, $+5.5 \mathrm{kV}$ ) to a high-resolution hybrid mass spectrometer $\left(\mathrm{TT}_{5600}{ }^{+}\right.$, ABSciex, Darmstadt, Germany) that combines a quadrupole for precursor ion selection with a time-of-fight (TOF) mass analyzer for product ion distinction. The mass spectrometer operated in the positive PIS mode after collision-induced dissociation (CID) with high sensitivity in the range from $\mathrm{m} / \mathrm{z} 50$ to $\mathrm{m} / \mathrm{z} 700$ for C(-HETE)P, propionylated-C(-HETE)P, herein referred to as PA-C(HETE)P, and the IS. The following MS settings were applied: declustering potential (DP) $60 \mathrm{~V}$, curtain gas (CUR) $30 \mathrm{psi}$ $\left(2.07 \times 10^{5} \mathrm{~Pa}\right)$, temperature $300{ }^{\circ} \mathrm{C}$, source gas $1(\mathrm{GS} 1)$ 50 psi $\left(3.45 \times 10^{5} \mathrm{~Pa}\right)$, source gas $2(\mathrm{GS} 2) 50$ psi $(3.45 \times$ $10^{5} \mathrm{~Pa}$ ), and collision energy (CE) $30 \mathrm{~V}$. The mass spectrometer was also connected with a calibrant delivery system (CDS, ABSciex) via an atmospheric pressure chemical ionization ion source inlet to infuse a calibrant solution ("APCI positive calibration solution," ABSciex) after each fifth $\mu \mathrm{LC}$ run with a flow of $500 \mu \mathrm{L} / \mathrm{min}$. The entire $\mu \mathrm{LC}$-ESI MS/ HRMS system was controlled by the Analyst TF 1.7.1 software (ABSciex) and the Eksigent control software (version $4.2)$.

\section{$\mu L C-E S I$ MS/MS analysis in PIS and SRM mode}

The presented procedure is based on the technique described by Blum et al. [20]. In brief, chromatography was carried out using a M5 microLC system comprising an integrated autosampler $\left(15^{\circ} \mathrm{C}\right)$ allowing $20 \mu \mathrm{L}$ sample volume injection (ABSciex), applying the same conditions of mobile and stationary phase as described above for $\mu \mathrm{LC}$-ESI MS/HRMS analysis. Using an ESI interface working in positive mode (OptiFlow turbo V source, $+5 \mathrm{kV}$ ) the QTrap $6500^{+}$mass spectrometer (ABSciex) was coupled to monitor product ions after CID of analytes using nitrogen as collision gas. The following MS settings were applied: DP $60 \mathrm{~V}$, CUR $30 \mathrm{psi}$ $\left(2.07 \times 10^{5} \mathrm{~Pa}\right)$, temperature $200{ }^{\circ} \mathrm{C}$, GS1 50 psi $(3.45 \times$ $\left.10^{5} \mathrm{~Pa}\right)$, GS2 $60 \mathrm{psi}\left(4.14 \times 10^{5} \mathrm{~Pa}\right)$, entrance potential $(\mathrm{EP})$ $10 \mathrm{~V}$, cell exit potential (CXP) $10 \mathrm{~V}$, and dwell time $50 \mathrm{~ms}$. The single protonated precursor ions of the biomarkers were fragmented with a $\mathrm{CE}$ of $30 \mathrm{~V}$ monitoring the following product ions for C(-HETE)P (m/z 323.1): m/z 105.1 (Qual 1) and $\mathrm{m} / \mathrm{z} 137.0$ (Qual 2), for carbobenzoxy-C(-HETE)P, herein referred to as Cbz-C(-HETE)P, (m/z 457.1): m/z 261.1 (Qual 1) and $\mathrm{m} / \mathrm{z}$ 105.0 (Qual 2) and PA-C(-HETE)P (m/z 379.1): $\mathrm{m} / \mathrm{z} 116.1$ (Qual 1), m/z 137.0 (Qual 2) and m/z 105.0 (Qual $3)$. The IS $d_{3}$-Atr was monitored with the product ions at $\mathrm{m} / \mathrm{z} 127.1$ (Qual 1) and m/z 93.1 (Qual 2) using a CE of $42 \mathrm{~V}$. For analysis in the PIS mode, product ions of the derivatized products were monitored under conditions described above in a mass range from $\mathrm{m} / \mathrm{z} 50$ to $\mathrm{m} / \mathrm{z} 500$. The entire $\mu$ LC-ESI MS/MS system was controlled by the Analyst TF 1.7.1 software (ABSciex) and the Eksigent control software (version 4.2).

\section{Characterization of the $\mu \mathrm{LC}$-ESI MS/MS (SRM) method for PA-C(-HETE)P}

\section{Selectivity}

The selectivity of the $\mu$ LC-ESI MS/MS (SRM) method was tested by analyzing prepared blank plasma derived from six individuals not exposed to SM but subjected to derivatization looking for any interference at the relevant retention time $\left(t_{R}\right)$ of PA-C(-HETE)P.

\section{Stability in the autosampler at $15{ }^{\circ} \mathrm{C}$}

The stability of PA-C(-HETE)P in a prepared plasma reference $(50 \mu \mathrm{M} \mathrm{SM})$ stored in the autosampler at $15^{\circ} \mathrm{C}$ was investigated by hourly analysis by $\mu$ LC-ESI MS/MS (SRM) 
over a period of $24 \mathrm{~h}$. Peak areas obtained from the XICs of Qual 1 as well as their corresponding peak area ratio to IS were used to follow the relative concentration-time profiles.

\section{Linear range, limit of detection, and limit of identification}

For elaboration of the linearity and to estimate the LOD and LOI, 12 plasma standards were produced by adding diluted solutions of SM in $\mathrm{iPrOH}$ to plasma yielding 12 different concentrations. SM solution $(20 \mu \mathrm{L})$ was added to $980 \mu \mathrm{L}$ plasma each $(\mathrm{n}=3)$ resulting in concentrations of $50 \mu \mathrm{M}$, $20 \mu \mathrm{M}, 4 \mu \mathrm{M}, 800 \mathrm{nM}, 400 \mathrm{nM}, 160 \mathrm{nM}, 80 \mathrm{nM}, 32 \mathrm{nM}$, $16 \mathrm{nM}, 6.4 \mathrm{nM}, 3.2 \mathrm{nM}$, and $1.28 \mathrm{nM}$. Standards were incubated, prepared, derivatized with propionic anhydride, and analyzed by $\mu$ LC-ESI MS/MS (SRM) following the standard protocol. Peak areas from the XICs of Qual 3 were plotted against the SM concentration for linear regression. The LOD was defined as the lowest concentration of SM still allowing the detection of PA-C(-HETE)P in all three replicates. The LOI was defined as the lowest concentration of SM, where the detection of PA-C(-HETE)P was possible in all three replicates still fitting the respective peak area ratios of the plasma reference $(50 \mu \mathrm{M} \mathrm{SM})$.

\section{Determination of the derivatization yield of PA-C(-HETE)P and $\mathrm{Cbz}-\mathrm{C}(-\mathrm{HETE}) \mathrm{P}$}

References derived from the incubation with $50 \mu \mathrm{M}$ and $12.5 \mu \mathrm{M}$ SM were prepared and proteolyzed in quadruplicate with pronase, derivatized either with propionic anhydride or BCF or they were not derivatized and analyzed afterwards following the standard protocol. Peak areas obtained from the XICs of the individual Qual 1 of C(-HETE)P, PA-C(-HETE)P, and Cbz-C(HETE)P were determined. The derivatization yield $(\omega)$ was calculated from the ratio of the C(-HETE)P peak area (A) after derivatization (a.d.) and without derivatization (w.d.) according to Eq. 1

$$
\begin{aligned}
\omega[\%]= & \left\{1-\mathrm{A}[\mathrm{C}(-\mathrm{HETE}) \mathrm{P}]_{\text {a.d. }} / \mathrm{A}[\mathrm{C}(-\mathrm{HETE}) \mathrm{P}]_{\text {w.d. }}\right\} \\
& \times 100
\end{aligned}
$$

As results obtained for both references $(12.5 \mu \mathrm{M}$ and $50 \mu \mathrm{M} \mathrm{SM}$ ) were identical, the mean and standard deviation (SD) were calculated.

\section{Determination of the response factors of PA-C(-HETE)P and $\mathrm{Cbz}-\mathrm{C}(\mathrm{HETE}) \mathrm{P}$}

Based on the same set of samples analyzed to determine $\omega$, the methodical response factor $\left(\mathrm{rf}_{\text {meth }}\right)$ was calculated as the ratio of the peak areas of Qual 1 of C(-HETE)P (w.d.) and peak areas of Qual 1 of the derivatized biomarker (a.d.) according to Eq. 2:

$\mathrm{rf}_{\text {meth }}=\mathrm{A}\{\mathrm{X}-\mathrm{C}(-\mathrm{HETE}) \mathrm{P}\}_{\text {a.d. }} / \mathrm{A}\{\mathrm{C}(-\mathrm{HETE}) \mathrm{P}\}_{\mathrm{w} . \mathrm{d}}$.

with $\mathrm{X}$ representing either $\mathrm{Cbz}$ or $\mathrm{PA}$.

The molecular response factor, $\mathrm{rf}_{\mathrm{mol}}$, was calculated according to Eq. 3:

$\mathrm{rf}_{\mathrm{mol}}=\mathrm{A}\{\mathrm{X}-\mathrm{C}(-\mathrm{HETE}) \mathrm{P}\}_{\text {a.d. }} /\left\{\mathrm{A}[\mathrm{C}(-\mathrm{HETE}) \mathrm{P}]_{\mathrm{w} \text {.d. }}-\mathrm{A}[\mathrm{C}(-\mathrm{HETE}) \mathrm{P}]_{\text {a.d }}\right\}$

with $\mathrm{X}$ representing either $\mathrm{Cbz}$ or PA.

As results obtained from both references $(12.5 \mu \mathrm{M}$ and $50 \mu \mathrm{M} \mathrm{SM}$ ) were identical, the mean and standard deviations (SD) were calculated for $\mathrm{rf}_{\text {meth }}$ and $\mathrm{rf}_{\text {mol }}$.

\section{Application of the novel procedure to real case plasma samples}

In 2015, seven individuals were supposed to be exposed to SM in the Middle East after a mortar attack [4]. Plasma samples were taken 15 days after the attack. At that time, four of the seven persons still suffered from skin lesions and respiratory problems. The other three persons did not show any symptoms neither a few hours after the attack nor 15 days after that [4]. Plasma samples obtained from the seven persons were prepared following the herein described standard protocol for derivatization with propionic anhydride and analyzed for the presence of PA-C(-HETE)P. Medically examined patients consented to subsequent blood sample analysis. Samples were made available in 2015 in an anonymous mode not allowing the investigator (Bundeswehr Institute of Pharmacology and Toxicology) the retrieval of personal identifying data of the donors.

\section{Results and discussion}

In general, the development of novel methods, the improvement of established procedures and the discovery of additional biomarkers are important efforts to extend the toolbox of methods for biomedical verification of SM exposure. The derivatization of biomarkers to generate novel diagnostic chemicals represents one of the relevant possibilities.

In the past decades, the derivatization of small molecules was often used to make, e.g., polar analytes more hydrophobic and thus suitable for GC analysis. Multiple reagents and methods were introduced as summarized by, e.g., Blau and Halket [21]. In contrast, methods to derivatize analytes for subsequent LC-MS analysis are less frequent. Several methods for the derivatization of proteins were introduced in the field of proteomics that allow the quantification of differently expressed proteins. Derivatization was done by, e.g., 
isotope labelling or metal coding [22-26]. In contrast, the derivatization of peptide biomarkers of CWA exposure is quite unusual. Merely, Chen et al. presented a procedure to convert C(-HETE)P with BCF [19]. However, the nonquantitative introduction of the hydrophobic carbobenzoxy moiety at the amine function of the N-terminus led to less favorable mass spectrometric and chromatographic properties. Therefore, an alternative derivatization procedure for a more favorable mass spectrometric behavior as well as a slighter increase of the hydrophobicity of the analyte appears as more beneficial.

Recently, van Faassen et al. reported on the propionylation of small molecules like tryptophan, serotonin, and 5hydroxytryptophan at the amine as well as at the hydroxyl groups under mildest conditions within 15 min, using propionic anhydride [27]. In general, the derivatization with propionic anhydride was also shown to be applicable to nucleotides, phenolic compounds, and cytokines, thus improving chromatographic separation and MS/MS responses [28-30]. Even though van Faassen et al. did not present an application for peptide derivatization, we transferred this rapid and simple procedure to the C(-HETE)P biomarker.

\section{Derivatization of C(-HETE)P with propionic anhydride}

Based on the results published by van Faasen et al. [27], the derivatization of the N-terminus as well as of the hydroxyl group of the HETE moiety of C(-HETE)P was assumed. Accordingly, $\mu$ LC-ESI MS/MS in the PIS mode was performed after derivatization to monitor product ions of the single protonated adduct C(-HETE)P (m/z 323.1) as well as of the single derivatized product potentially present as PA-C(HETE)P or C(-HETE-PA)P ( $\mathrm{m} / \mathrm{z}$ 379.1) and of the twofold derivatized biomarker PA-C(-HETE-PA)P (m/z 436.2). After the addition of $10 \mu \mathrm{L}$ propionic anhydride, the XIC of Qual 1 of C(-HETE)P showed no peak anymore (data not shown), thus proving quantitative derivatization of the dipeptide. In addition, only one peak of a single derivatized product was observed at $t_{R} 9.7 \mathrm{~min}$, thus demonstrating the selective derivatization at only one functional group of C(-HETE)P. The corresponding product ion spectrum extracted from the $\mu \mathrm{LC}$ ESI MS/MS PIS run is shown in Fig. 1.

The product ion at $\mathrm{m} / \mathrm{z} 116.1$ belonged to the proline residue and the ion at $\mathrm{m} / \mathrm{z} 70.0$ represented the immonium ion of proline. The presence of the product ions at $\mathrm{m} / \mathrm{z} 105.0$ (HETE moiety) and at $\mathrm{m} / \mathrm{z} 137.0$ (HETE moiety plus sulfur atom of the $\mathrm{C}^{34}$ residue) strongly supported that the derivatization happened at the N-terminus of the $\mathrm{C}^{34}$ residue and not at the hydroxyl function of the HETE moiety as illustrated in Fig. 2. The latter derivative might have resulted in product ions at $\mathrm{m} / \mathrm{z} 161.1$ (PA-HETE moiety) and at $\mathrm{m} / \mathrm{z} 193.1$ (PAHETE plus sulfur atom of $\mathrm{C}^{34}$ ). None of these ions was detected.

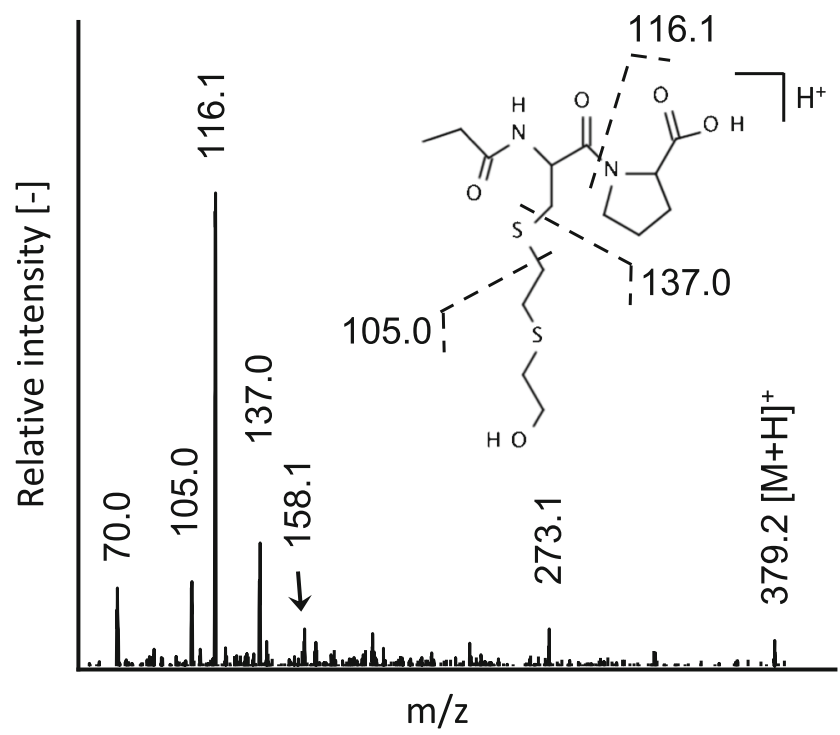

Fig. 1 Product ion spectrum of single protonated PA-C(-HETE)P. The single protonated propionylated (PA) biomarker was subjected to collision-induced dissociation $\left([\mathrm{M}+\mathrm{H}]^{+}, \mathrm{m} / \mathrm{z} 379.1\right)$ to extract the MS/ MS data from a $\mu$ LC-ESI MS/MS (PIS) run. Cleavage sites resulting in product ions used for the $\mu \mathrm{LC}$-ESI MS/MS (SRM) method are depicted

In addition, no peak was found for a potentially twofold derivatized biomarker, thus underlining the selectivity of the derivatization at the $\mathrm{N}$-terminus. The same exclusive selectivity was found even though applying a larger molar excess of propionic anhydride (125 $\mu \mathrm{L}$ corresponding to a molar excess of $1.5 \times 10^{6}$ ) in the presence of triethylamine (TEA) as a strong base. These findings are in agreement with other studies showing that the propionylation of primary aliphatic hydroxyl groups often requires either large bases like imidazole or non-aqueous solvents or propionic anhydride as the solvent itself [30,31]. Hence, the hydroxyl group of the HETE moiety was obviously not reactive under the conditions applied.

The structural assignment of product ions was confirmed by MS/HRMS coupled to $\mu \mathrm{LC}$ as summarized in Table 1 . The product ion at $\mathrm{m} / \mathrm{z} 116.071$, corresponding to the $y_{1}$ fragment of the peptide backbone, was the most abundant product ion. This product ion was also observed after CID of C(-HETE)P as described by John et al. [16], but it was found at much lower relative intensity. The favored dissociation site at the proline amide bond of the PA derivative may be explained by the preferred site of protonation during the ESI process. In the non-derivatized C(-HETE)P molecule, the free amine group is the most favorable site of protonation. The protonated $\mathrm{N}$-terminus enhances the energy, thus favoring the cleavage of the positively charged HETE moiety (m/z 105.037, Table 1$)$. In contrast, the propionylated N-terminus of PA-C(-HETE)P is less favorable for protonation due to its lower basicity. Therefore, the tertiary amine group of the proline residue might be protonated primarily, thus resulting in the cleavage of the amide bond of proline as the predominant fragmentation site. In addition, the product ions at $\mathrm{m} / \mathrm{z} 137.009$ and at 
Fig. 2 Derivatization procedure of C(-HETE)P with propionic anhydride

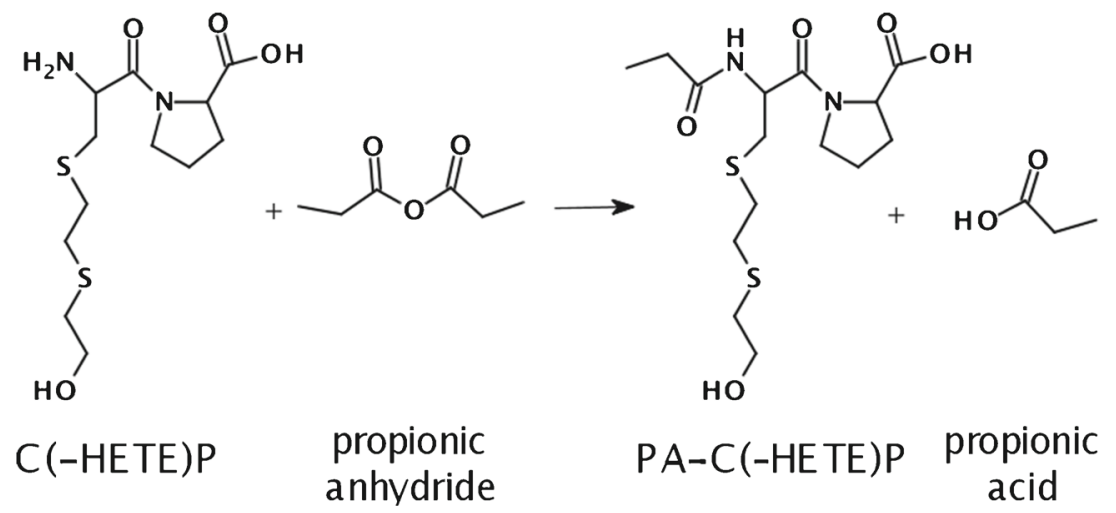

HETE: hydroxyethylthioethyl

$\mathrm{m} / \mathrm{z}$ 105.0373, which are characteristic for the HETE moiety, were also detected (Table 1). Accordingly, product ions at m/z 116.1 (Qual 1), m/z 137.0 (Qual 2), and m/z 105.0 (Qual 3 ) were used to set up the $\mu \mathrm{LC}$-ESI MS/MS (SRM) method. Exemplarily, the XIC of Qual 3 of a standard ( $4 \mu \mathrm{M} \mathrm{SM})$ is shown in Fig. $3 b$.

\section{Optimization of derivatization conditions}

The time for derivatization was varied by stopping the reaction with added water after various periods causing the hydrolysis of remaining propionic anhydride to propionic acid. It was found that quantitative conversion of C(-HETE)P has already happened after $10 \mathrm{~s}$ after the addition of $10 \mu \mathrm{L}$ propionic anhydride. Lower volumes of the reagent did not allow quantitative conversion despite prolonged reaction times. Accordingly, the standard protocol made use of $10 \mu \mathrm{L}$ propionic anhydride reacting for $10 \mathrm{~s}$ at $\mathrm{RT}$.

\section{Derivatization of C(-HETE)P with BCF}

C(-HETE)P was derivatized with BCF according to the published protocol of Chen et al. yielding Cbz-C(-HETE)P [19]. Accordingly, this derivative was monitored by the product ions at $\mathrm{m} / \mathrm{z} 261.1$ (Qual 1) and m/z 105.0 (Qual 2) in $\mu \mathrm{LC}$ ESI MS/MS (SRM) analysis ( $\left.t_{R} 13.2 \mathrm{~min}\right)$. In our study, the yield was found to be $92.8 \%$. Surprisingly, this yield was significantly higher than that originally reported by Chen et al. $(47 \%)$ [19].

\section{Determination of the derivatization yield}

The derivatization of C(-HETE)P with propionic anhydride was quantitative and therefore slightly better than the reaction with BCF. For both reagents, calculations of $\omega$ showed identical results independent of the concentrations of C(-HETE)P that were derived from references $(50 \mu \mathrm{M}$ and $12.5 \mu \mathrm{M} \mathrm{SM})$.
Accordingly, the mean of $\omega$ for PA-C(-HETE)P was $100 \% \pm$ $0 \%$ and that of Cbz-C(-HETE)P $92.8 \% \pm 2.0 \%$ (Table 2).

\section{Characterization of the $\mu$ LC-ESI MS/MS (SRM) proce- dure for PA-C(-HETE)P}

\section{Selectivity}

Following the standard protocol of sample preparation including two steps of protein precipitation, the reliable detection of PA-C(-HETE)P was possible without any interferences at $t_{R}$ $9.7 \mathrm{~min}$. Exemplarily, the XIC of the ion at $\mathrm{m} / \mathrm{z} 105.0$ (Qual 3) is illustrated in Fig. 3a.

\section{Stability in the autosampler at $15^{\circ} \mathrm{C}$}

The peak areas of the XIC of Qual 1 of PA-C(-HETE)P were found to be constant (relative standard deviation, RSD: $2 \%$ ) over the entire test period of $24 \mathrm{~h}$. Peak area ratios of PA-C(-HETE)P to $\mathrm{d}_{3}$-Atr used as IS remained constant as well (RSD: $3 \%$ ) thus documenting no time-dependent decomposition of the derivative. Furthermore, $t_{R}$ of PA-C(-HETE)P remained constant at $9.7 \mathrm{~min} \pm 0.1 \mathrm{~min}$ not showing any shift, thus documenting robust and reliable chromatography. The high stability is highly beneficial when analyzing larger sets of samples.

\section{Linearity, limit of detection, and limit of identification}

The peak areas of PA-C(-HETE)P showed excellent linear relationship to the concentration of $\mathrm{SM}$ in plasma in a range from $32 \mathrm{nM}$ to $50 \mu \mathrm{M}$ (Qual 3), thus covering concentrations of toxicological relevance. The linearity documented the reproducibility of the derivatization procedure and its applicability to a broad concentration range of C(-HETE)P. The LOD for PA-C(-HETE)P was determined to be $32 \mathrm{nM}$.

Based on quadruplicate analysis of references, ion ratios (peak area ratios) of the different product ions were calculated yielding $26.5 \% \pm 0.2 \%$ for Qual 2/Qual 1 and $18.6 \% \pm 0.1 \%$ 
Table 1 Product ions of single protonated PA-C(-HETE)P

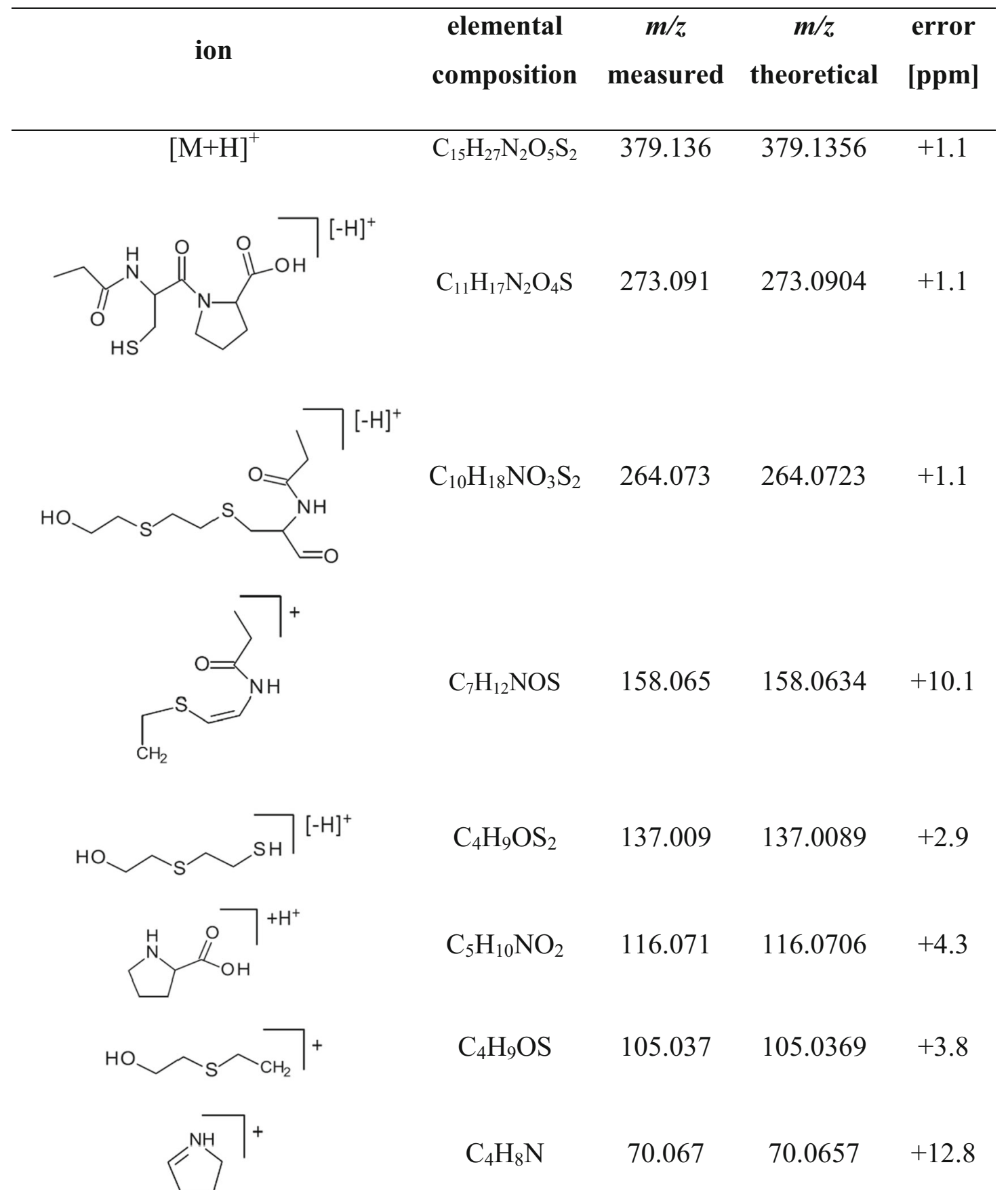

Data was extracted from a $\mu$ LC-ESI MS/HRMS run of the HETE-HSA adduct after enzymatic cleavage with pronase and subsequent propionylation (PA) monitoring product ions of $\mathrm{m} / \mathrm{z} 379.1$. This reference was produced by incubation of plasma with SM (50 $\mu \mathrm{M})$. The structures represent one possible isomer each

for Qual 3/Qual 1. At least one of these ratios should be met by any sample or standard defining the LOI [32]. Accordingly, the LOI for PA-C(-HETE)P was found at $160 \mathrm{nM}$ based on the ratio of Qual 3/Qual 1. The corresponding XIC of Qual 3 is shown in Fig. 3c.

\section{Determination of $\mathbf{r f}_{\text {meth }}$ and $\mathbf{r} \mathbf{f}_{\text {mol }}$}

The relevant measures to characterize a novel biomarker for forensic trace analysis are usually the LOD and LOI. Accordingly, we determined both values for PA-C(HETE)P. 


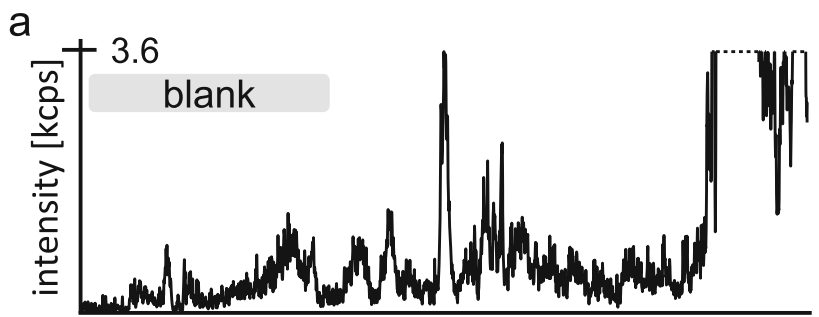

b

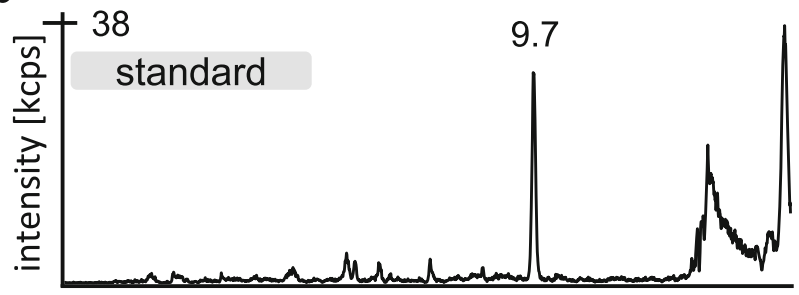

C

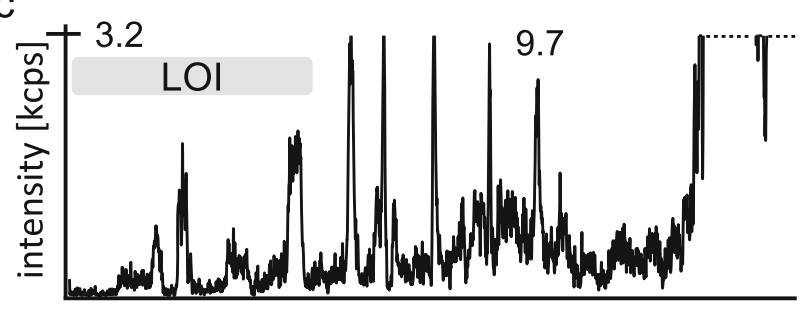

d

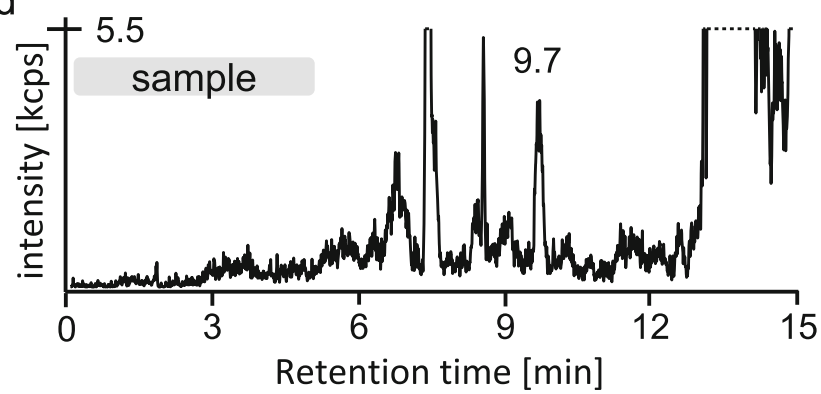

Fig. 3 Extracted ion chromatograms of the derivatized biomarker of SM exposure PA-C(-HETE)P in human plasma. a Blank plasma not exposed to $\mathrm{SM}$, b standard produced after plasma incubation with $4 \mu \mathrm{M}$ SM, c plasma incubated with SM (160 nM) corresponding to the lower limit of identification (LOI), and $\mathbf{d}$ sample of a patient poisoned with SM in the Middle East [4]. The samples were prepared by pronase cleavage of adducted HSA in plasma and subsequent derivatization with propionic anhydride. Analyses were performed by $\mu L C$-ESI MS/MS (SRM). For reasons of clarity, only the product ion trace of m/z 105.0 is shown

However, these values are highly dependent on multiple factors like matrices, the sample preparation, working and dilution steps, and the mass spectrometer used. Therefore, the comparison of LOD and LOI is not the only way to evaluate the suitability of biomarkers. We herein introduce the $\mathrm{rf}_{\text {meth }}$ and $\mathrm{rf}_{\mathrm{mol}}$ values which address a relative mass spectrometric response as an independent measure. The $\mathrm{rf}_{\text {meth }}$ value characterizes the relative signal yield (peak area) of the derivatized biomarker compared to the signal yield (peak area) of the nonderivatized biomarker. This value provides a measure of the procedure-dependent signal yield. In contrast, the $\mathrm{rf}_{\text {mol }}$ value
Table 2 Methodical and molecular response factors and yield of PAC(-HETE)P and Cbz-C(-HETE)P

\begin{tabular}{llll}
\hline Analyte & $\omega[\%]$ & $\mathrm{rf}_{\text {meth }}[-]$ & $\mathrm{rf}_{\text {mol }}[-]$ \\
\hline Cbz-C(-HETE)P & $92.8 \pm 2.0$ & $0.15 \pm 0.03$ & $0.16 \pm 0.03$ \\
PA-C(-HETE)P & $100 \pm 0$ & $0.37 \pm 0.07$ & $0.37 \pm 0.07$ \\
\hline
\end{tabular}

Data characterize the derivatization of C(-HETE)P with propionic anhydride yielding PA-C(-HETE)P and with benzyl chloroformate yielding Cbz-C(-HETE)P

The rf values represent the mean and standard deviation of results obtained from plasma references incubated with $50 \mu \mathrm{M}$ and $12.5 \mu \mathrm{M} \mathrm{SM}(\mathrm{n}=$ 4, each). Values were calculated according to Eqs. 1-3

$\omega$ yield of derivatization, $r f_{\text {meth }}$ methodical response factor, $r f_{\text {mol }}$ molecular response factor, $\mathrm{Cbz}$ carbobenzoxy, $P A$ propionyl

characterizes the relative signal yield (peak area) of the derivatized molecule considering the derivatization yield.

For PA-C(-HETE)P, $\mathrm{rf}_{\text {meth }}$ and $\mathrm{rf}_{\text {mol }}$ were both found to be 0.37 , thus documenting a lower signal yield than obtained for non-derivatized C(-HETE)P (Table 2). This might be due to the modified N-terminus of PA-C(-HETE)P, which protonation is less favorable than that of the free amine group of $\mathrm{C}$ (-HETE)P, thus reducing the ionization efficacy. However, $\mathrm{rf}_{\text {meth }}$ and $\mathrm{rf}_{\text {mol }}$ of PA-C(-HETE)P were more than two times higher than those of Cbz-C(-HETE)P found to be as low as about 0.16 (Table 2). Therefore, the propionylated derivative exhibits reasonably improved mass spectrometric properties when compared to the Cbz derivative. Even though the mass spectrometric response is lower than that of the nonderivatized biomarker, PA-C(-HETE)P might be valuable with respect to its different $t_{R}$ if donor-specific plasma-derived interferences deteriorate C(-HETE)P analysis.

\section{Application of the novel procedure to real case samples}

The $\mu$ LC-ESI MS/MS (SRM) method introduced herein was applied to plasma samples obtained from victims of SM poisoning. As reported quite recently by our group, the SM exposure of four out of seven persons was proven by the detection of diverse HSA-derived HETE adducts including, e.g., C(-HETE)P and C(-HETE)PF [4]. The same set of samples was analyzed in the present study to monitor PA-C(-HETE)P. In accordance with our earlier report, poisoning by SM was detected only in the plasma of the four persons who also showed clinical signs and symptoms. As an example, the chromatogram of one sample is shown in Fig. 3d. The congruency of our novel and earlier data document that the procedure for derivatization with propionic anhydride is applicable to plasma samples from real exposure scenarios and provides reliable results. Even though sample draw in the Middle East was done 15 days after exposure [4], the remaining HSA 
adduct concentrations were sufficient to be traceable by the PA-C(-HETE)P biomarker.

\section{Conclusions}

We herein present a plasma sample preparation procedure and bioanalytical $\mu$ LC-ESI MS/MS (SRM) method for the precolumn derivatization of the dipeptide C(-HETE)P and the selective and sensitive detection of the derivative. The procedure was proven to be suitable for analysis of samples from real cases of SM poisoning. A simple split of the prepared sample that is ready for the detection of C(-HETE)P, and its subjection to the rapid and quantitative derivatization, may enable economic and fast monitoring of both markers without the need for an additional proteolytic step as it would be needed for C(-HETE)PF. The $t_{R}$ of PA-C(-HETE)P (9.7 min) is significantly different from that of C(-HETE)P $\left(t_{R} 4.6 \mathrm{~min}\right)$ and can therefore provide a valuable alternative if any sample shows matrix-derived interferences hindering $\mathrm{C}($-HETE)P monitoring. Therefore, we suggest this derivative as a novel biomarker in addition to the already internationally accepted non-derivatized peptide C(-HETE)P.

\begin{abstract}
Abbreviations A, Peak area; $A C N$, Acetonitrile; a.d., After derivatization; $B C F$, Benzyl chloroformate; $C b z$, Carbobenzoxy moiety; $C E$, Collision energy; $C I D$, Collision-induced dissociation; $C U R$, Curtain gas; $C W A$, Chemical warfare agent; $C W C$, Chemical Weapons Convention; $C X P$, Cell exit potential; $d_{3}$-Atr, Three-fold deuterated atropine; $D P$, Declustering potential; EDTA, Ethylenediaminetetraacetic acid; $E P$, Entrance potential; $E S I$, Electrospray ionization; $F A$, Formic acid; GS1, Source gas 1; GS2, Source gas 2; HETE, Hydroxyethylthioethyl moiety; HSA, Human serum albumin; $i \mathrm{PrOH}$, Isopropanol; IS, Internal standard; $L O D$, Limit of detection; $L O I$, Limit of identification; $\mu L C$, Microliquid chromatography; $M S / M S$, Tandem-mass spectrometry; $M S$ / $H R M S$, High-resolution tandem-mass spectrometry; $N M R$, Nuclear magnetic resonance; $O P C W$, Organisation for the Prohibition of Chemical Weapons; $P A$, Propionyl moiety; $P B S$, Phosphate-buffered saline; $P I S$, Product ion scan; Qual, Qualifying ion; RT, Room temperature; $S M$, Sulfur mustard; $S R M$, Selected reaction monitoring; $T D G$, Thiodiglycol; $T E A$, Triethylamine; TFA, Trifluoroacetic acid; TIC, Total ion chromatogram; $t_{R}$, Retention time; $w . d$., Without derivatization; $\omega$, Derivatization yield; $X I C$, Extracted ion chromatogram
\end{abstract}

Funding Open Access funding enabled and organized by Projekt DEAL. Part of the work was supported by the German Research Foundation (Deutsche Forschungsgemeinschaft, DFG, Grant/Award Number Research Training Group GRK 2338).

\section{Declarations}

Informed consent Medically examined patients consented to blood sample drawing and diagnostic analysis.

Conflict of interest The authors declare no competing interests.
Open Access This article is licensed under a Creative Commons Attribution 4.0 International License, which permits use, sharing, adaptation, distribution and reproduction in any medium or format, as long as you give appropriate credit to the original author(s) and the source, provide a link to the Creative Commons licence, and indicate if changes were made. The images or other third party material in this article are included in the article's Creative Commons licence, unless indicated otherwise in a credit line to the material. If material is not included in the article's Creative Commons licence and your intended use is not permitted by statutory regulation or exceeds the permitted use, you will need to obtain permission directly from the copyright holder. To view a copy of this licence, visit http://creativecommons.org/licenses/by/4.0/.

\section{References}

1. Kehe K, Szinicz L. Medical aspects of sulphur mustard poisoning. Toxicology. 2005;214:198-209.

2. Evison D, Hinsley D, Rice P. Chemical weapons. BMJ. 2002;324: 332.

3. Quillen C. The Islamic State's evolving chemical arsenal. Studies in Conflict \& Terrorism. 2016;39:1019-30. https://doi.org/10.1080/ 1057610X.2016.1154364.

4. John H, Koller M, Worek F, Thiermann H, Siegert M. Forensic evidence of sulfur mustard exposure in real cases of human poisoning by detection of diverse albumin-derived protein adducts. Arch Toxicol. 2019;93:1881-91.

5. Sezigen S, Ivelik K, Ortatatli M, Almacioglu M, Demirkasimoglu M, Eyison RK, et al. Victims of chemical terrorism, a family of four who were exposed to sulfur mustard. Toxicol Lett. 2019;303:9-15.

6. Bullman T, Kang H. A fifty year mortality follow-up study of veterans exposed to low level chemical warfare agent, mustard gas. Ann Epidemiol. 2000;10:333-8.

7. Blum M-M, Mamidanna RVSM. Analytical chemistry and the Chemical Weapons Convention. Anal Bioanal Chem. 2014;406: 5067-9. https://doi.org/10.1007/s00216-014-7931-4.

8. John H, van der Schans MJ, Koller M, Spruit HET, Worek F, Thiermann H, et al. Fatal sarin poisoning in Syria 2013: forensic verification within an international laboratory network. Forensic Toxicol. 2018;36:61-71.

9. Steinritz D, Striepling E, Rudolf K-D, Schröder-Kraft C, Püschel K, Hullard-Pulstinger A, et al. Medical documentation, bioanalytical evidence of an accidental human exposure to sulfur mustard and general therapy recommendations. Toxicol Lett. 2016;244:112-20.

10. Xu H, Nie Z, Zhang Y, Li C, Yue L, Yang W, et al. Four sulfur mustard exposure cases: overall analysis of four types of biomarkers in clinical samples provides positive implication for early diagnosis and treatment monitoring. Toxicol Rep. 2014;1:533-43.

11. Noort D, Hulst AG, Trap HC, de Jong LP, Benschop HP. Synthesis and mass spectrometric identification of the major amino acid adducts formed between Sulphur mustard and haemoglobin in human blood. Arch Toxicol. 1997;71:171-8.

12. Noort D, Fidder A, Hulst AG, de Jong LPA, Benschop HP. Diagnosis and dosimetry of exposure to sulfur mustard: development of a standard operating procedure for mass spectrometric analysis of haemoglobin adducts: exploratory research on albumin and keratin adducts. J Appl Toxicol. 2000;20:S187-S92.

13. Zubel T, Hochgesand S, John H, Steinritz D, Schmidt A, Bürkle A, et al. A mass spectrometric platform for the quantitation of sulfur mustard-induced nucleic acid adducts as mechanistically relevant biomarkers of exposure. Arch Toxicol. 2019;93:61-79.

14. Noort D, Hulst AG, de Jong LPA, Benschop HP. Alkylation of human serum albumin by sulfur mustard in vitro and in vivo: mass 
spectrometric analysis of a cysteine adduct as a sensitive biomarker of exposure. Chem Res Toxicol. 1999;12:715-21.

15. Benschop HP, van der Schans GP, Noort D, Fidder A, MarsGroenendijk RH, de Jong LPA. Verification of exposure to sulfur mustard in two casualties of the Iran-Iraq conflict. J Anal Toxicol. 1997;21:249-51. https://doi.org/10.1093/jat/21.4.249.

16. John H, Siegert M, Gandor F, Gawlik M, Kranawetvogl A, Karaghiosoff K, et al. Optimized verification method for detection of an albumin-sulfur mustard adduct at Cys 34 using a hybrid quadrupole time-of-flight tandem mass spectrometer after direct plasma proteolysis. Toxicol Lett. 2016;244:103-11.

17. Gandor F, Gawlik M, Thiermann H, John H. Evidence of sulfur mustard exposure in human plasma by LC-ESI-MS-MS detection of the albumin-derived alkylated HETE-CP dipeptide and chromatographic investigation of its cis/trans isomerism. J Anal Toxicol. 2015;39:270-9.

18. John H, Willoh S, Hörmann P, Siegert M, Vondran A, Thiermann $\mathrm{H}$. Procedures for analysis of dried plasma using microsampling devices to detect sulfur mustard albumin adducts for verification of poisoning. Anal Chem. 2016;88:8787-94.

19. Chen B, Yu H, Liu S, Liu C, Liang L, Li X, et al. A sensitive quantification approach for detection of HETE-CP adduct after benzyl chloroformate derivatization using ultra-high-pressure liquid chromatography tandem mass spectrometry. Anal Bioanal Chem. 2019;411:3405-15.

20. Blum MM, Richter A, Siegert M, Thiermann H, John H. Adduct of the blistering warfare agent sesquimustard with human serum albumin and its mass spectrometric identification for biomedical verification of exposure. Anal Bioanal Chem. 2020;412:7723-37.

21. Blau K, Halket JM, editors. Handbook of derivatives for chromatography. 2nd ed. Chichester: John Wiley \& Sons; 1993.

22. Sethuraman M, McComb ME, Huang H, Huang S, Heibeck T, Costello CE, et al. Isotope-coded affinity tag (ICAT) approach to redox proteomics: identification and quantitation of oxidantsensitive cysteine thiols in complex protein mixtures. J Proteome Res. 2004;3:1228-33.

23. Choe L, D'Ascenzo M, Relkin NR, Pappin D, Ross P, Williamson $\mathrm{B}$, et al. 8-Plex quantitation of changes in cerebrospinal fluid protein expression in subjects undergoing intravenous immunoglobulin treatment for Alzheimer's disease. Proteomics. 2007;7:3651-60.
24. Ross PL, Huang YN, Marchese JN, Williamson B, Parker K, Hattan $\mathrm{S}$, et al. Multiplexed protein quantitation in Saccharomyces cerevisiae using amine-reactive isobaric tagging reagents. Mol Cell Proteomics. 2004;3:1154-69. https://doi.org/10.1074/mcp.M400129-MCP200.

25. Schwarz G, Beck S, Weller MG, Linscheid MW. MeCAT-new iodoacetamide reagents for metal labeling of proteins and peptides. Anal Bioanal Chem. 2011;401:1203.

26. Schmidt A, Kellermann J, Lottspeich F. A novel strategy for quantitative proteomics using isotope-coded protein labels. Proteomics. 2005;5:4-15.

27. van Faassen M, Bouma G, de Hosson LD, Peters MAM, KatsUgurlu G, de Vries EGE, et al. Quantitative profiling of plateletrich plasma indole markers by direct-matrix derivatization combined with LC-MS/MS in patients with neuroendocrine tumors. Clin Chem. 2019;65:1388-96.

28. Nordström A, Tarkowski P, Tarkowska D, Dolezal K, Åstot C, Sandberg G, et al. Derivatization for LC-electrospray ionizationMS: a tool for improving reversed-phase separation and ESI responses of bases, ribosides, and intact nucleotides. Anal Chem. 2004;76:2869-77.

29. Pierce WM Jr, Nerland DE. Qualitative and quantitative analyses of phenol, phenylglucuronide, and phenylsulfate in urine and plasma by gas chromatography/mass spectrometry. J Anal Toxicol. 1988;12:344-7.

30. Escrig-Doménech A, Simó-Alfonso EF, Herrero-Martínez JM, Ramis-Ramos G. Derivatization of hydroxyl functional groups for liquid chromatography and capillary electroseparation. J Chromatogr A. 2013;1296:140-56.

31. Brindaba CR, Dey SS, Hajra A. Highly efficient acylation of alcohols, amines and thiols undersolvent-free and catalyst-free conditions. Green Chem. 2003;5:44-6.

32. Work instruction for the reporting of the results of the OPCW biomedical proficiency tests. OPCW; 2020, https://www.opcw.org/ sites/default/files/documents/S_series/2017/en/s-1515-2017_e_. pdf, Accessed 02.12.2020.

Publisher's note Springer Nature remains neutral with regard to jurisdictional claims in published maps and institutional affiliations. 